\title{
AHIF promotes glioblastoma progression and radioresistance via exosomes
}

\author{
XUEJUN DAI ${ }^{1 *}$, KEMAN LIAO $^{2 *}$, ZHIJUN ZHUANG $^{1}$, BINGHONG CHEN $^{2}$, ZHIYI ZHOU $^{2}$, \\ SUNHAI ZHOU ${ }^{2}$, GUOSHI LIN ${ }^{1}$, FEIFEI ZHANG ${ }^{1}$, YINGYING LIN ${ }^{2}$, YIFENG MIAO ${ }^{2}$, \\ ZHIQIANG $\mathrm{LI}^{3}$, RENHUA HUANG ${ }^{4}$, YONGMING QIU ${ }^{2}$ and RUISHENG LIN ${ }^{1 *}$
}

${ }^{1}$ Department of Neurosurgery, Zhangzhou Affiliated Hospital of Fujian Medical University, Zhangzhou, Fujian 363000; ${ }^{2}$ Department of Neurosurgery, Renji Hospital, School of Medicine, Shanghai Jiaotong University, Shanghai 200120; ${ }^{3}$ Shanghai Neurological Research Institute of Anhui University of Science and Technology, Shanghai Fengxian District Central Hospital, Shanghai 201499; ${ }^{4}$ Department of Radiation Oncology,

Renji Hospital, School of Medicine, Shanghai Jiaotong University, Shanghai 200120, P.R. China

Received June 21, 2018; Accepted October 11, 2018

DOI: 10.3892/ijo.2018.4621

\begin{abstract}
Glioblastoma multiforme (GBM) has the highest mortality rate among patients with brain tumors, and radiotherapy forms an important part of its treatment. Thus, there is an urgent requirement to elucidate the mechanisms conferring GBM progression and radioresistance. In the present study, it was identified that antisense transcript of hypoxia-inducible factor- $1 \alpha$ (AHIF) was significantly upregulated in GBM cancerous tissues, as well as in radioresistant GBM cells. The expression of AHIF was also upregulated in response to radiation. Knockdown of AHIF in GBM cells decreased viability and invasive capacities, and increased the proportion of apoptotic cells. By contrast, overexpression of AHIF in GBM cells increased viability and invasive capacities, and decreased the proportion of apoptotic cells. Furthermore, exosomes derived from AHIF-knockdown GBM cells inhibited viability, invasion and radioresistance, whereas exosomes derived from AHIF-overexpressing GBM cells promoted viability, invasion and radioresistance. Further biochemical analysis identified that AHIF regulates factors associated with migration and angiogenesis in exosomes. To the best of our knowledge, the present study is the first to establish that AHIF promotes glioblastoma progression and
\end{abstract}

Correspondence to: Professor Ruisheng Lin, Department of Neurosurgery, Zhangzhou Affiliated Hospital of Fujian Medical University, 59 West Shengli Road, Zhangzhou, Fujian 363000, P.R. China

E-mail: linzzsyy@126.com

*Contributed equally

Key words: glioblastoma, antisense transcript of hypoxia-inducible factor $1 \alpha$, exosome, progression, radioresistance radioresistance via exosomes, which suggests that AHIF is a potential therapeutic target for GBM.

\section{Introduction}

Glioblastoma multiforme (GBM) is the most frequently diagnosed and lethal type of primary brain tumor, and is characterized by high invasive ability. Although surgery is the primary treatment strategy for GBM, extensive diffuse parenchymal invasion often results in failure of surgical resection (1-3). Therefore, radiotherapy is a major adjuvant therapy for patients with GBM (4). It has long been recognized that GBM tumors are heterogeneous in their radiation response, and the degree of radiosensitivity is thought to be associated with intrinsic and extrinsic properties of the tumor cell population (5-7). The effects and underlying molecular mechanisms of GBM progression and radioresistance have yet to be clarified.

Long non-coding RNAs (lncRNAs) are non-protein-coding transcripts longer than 200 nucleotides. Accumulating evidence has indicated that certain lncRNAs serve important functions in the regulation of various biological processes, including proliferation, differentiation and cell death (8-14). The lncRNA AHIF is the natural antisense transcript of hypoxia-inducible factor- $1 \alpha$ (HIF- $1 \alpha)$, and is exactly complementary to the 3'-untranslated region of HIF-1 $\alpha$ mRNA $(15,16)$. A small number of studies have addressed the function of AHIF in tumor progression (17-20). The expression of AHIF was detected in invasive ductal carcinoma samples, whereas adjacent non-cancer tissues did not exhibit AHIF expression. AHIF is a poor prognostic marker in breast cancer contributing in HIF-1 $\alpha$ mRNA regulation (18).

Exosomes are nano-sized membrane vesicles with diameters between 30 and $100 \mathrm{~nm}$ (21-23). It has previously been reported that cancer-associated exosomes serve important roles in regulating the cellular functions of cancerous cells, fibroblasts, vascular smooth muscle cells and endothelial cells through effectively delivering microRNAs, mRNAs and 
proteins (24-29). However, the functions of exosomes in GBM progression and radiotherapy remain unknown.

In the present study, the reverse transcription-quantitative polymerase chain reaction ( $\mathrm{RT}-\mathrm{qPCR}$ ), was used to identify the expression of AHIF in GBM cancerous tissues and radioresistant GBM cells. Functional experiments in vitro were performed to address the hypothesis that AHIF could promote glioblastoma progression and radioresistance via exosomes. Further biochemical analysis identified that AHIF regulates factors associated with migration and angiogenesis in exosomes. To the best of our knowledge, the present study is the first to establish that AHIF promotes glioblastoma progression and radioresistance via exosomes, which may be a potential therapeutic target.

\section{Materials and methods}

Patients and tissue samples. The present study was approved by the Ethics Committee of Renji Hospital, School of Medicine, Shanghai Jiao Tong University (Shanghai, China). Written informed consent was obtained from patients for participation in the study. A total of 31 patients (including 16 males and 15 females) with histologically confirmed GBM were recruited at Renji Hospital between January 2016 and December 2017 for inclusion in the present study. The mean age of patients was $49.38 \pm 15.87$ years (range, $13-85$ years). Adjacent normal tissues were also collected from 7 of the patients with GBM.

Cell culture. The human GBM cell lines U87-MG (glioblastoma of unknown origin; the cell line was authenticated by short tandem repeat profiling), U251-MG, A172 and T98G (purchased in 2014 from the Cell Bank of the Chinese Academy of Sciences, Shanghai, China) were cultured in Dulbecco's modified Eagle's medium (Hyclone; GE Healthcare, Logan, UT, USA) supplemented with $10 \%$ fetal bovine serum (FBS; Gibco; Thermo Fisher Scientific, Inc., Waltham, MA, USA) and maintained in a humidified atmosphere at $37^{\circ} \mathrm{C}$ with $5 \% \mathrm{CO}_{2}$.

Radiation treatment. Cells [U87-MG and U251-MG, as well as respective AHIF-knockdown (KD) and AHIF-overexpression (OE) cells] in culture were treated with an irradiator (GE3000) using a ${ }^{137} \mathrm{Cs}$ source at a dose rate of $4.0 \mathrm{~Gy} / \mathrm{min}$ for $90 \mathrm{sec}$. During irradiation, the cultures were maintained in the cell culture incubator $\left(5 \% \mathrm{CO}_{2}\right.$ at $\left.37^{\circ} \mathrm{C}\right)$.

$R T$ - $q P C R$. RNA extraction, cDNA synthesis and RT-qPCR were performed as described previously (20). Total RNA was extracted from tissues and/or cells using TRIzol ${ }^{\circledR}$ (Invitrogen; Thermo Fisher Scientific, Inc.). A total of $1 \mu \mathrm{g}$ RNA was used for first-strand cDNA synthesis $\left(99^{\circ} \mathrm{C}\right.$ for 5 min and $42^{\circ} \mathrm{C}$ for $45 \mathrm{~min}$ ) using an oligo-dT primer and $\mathrm{M}$-myeloblastosis virus reverse transcriptase XL (Promega Corporation, Madison, WI, USA). The synthesized first-strand cDNA was used for each qPCR. The qPCR primers were as follows: Human AGIF forward, 5'-TCAACATACATTAAGGTGATGGCAC-3' and reverse, 5'-ATTTGCTTCAACACCTCCAACTC-3'; human vascular endothelial growth factor A (VEGF-A) forward, 5'-TTGCCTTGCTGCTCTACCTCCA-3' and reverse, 5'-GAT GGCAGTAGCTGCGCTGATA-3'; human angiogenin forward, 5'-CAACAAGCGCAGCATCAAG-3' and reverse,
5'-CAAGTGGTGACCTGGAAAGAAG-3'. SYBR-Green PCR Master Mix (Applied Biosystems; Thermo Fisher Scientific, Inc.) was used for the qPCR experiments. $\beta$-actin was used as an internal control. The relative expression of target genes was determined using the $2^{-\Delta \Delta \mathrm{Cq}}$ method (30). The qPCR primers for $\beta$-actin were: Forward, 5'-CACCATTGG CAATGAGCGGTTC-3' and reverse, 5'-AGGTCTTTGCGG ATGTCCACGT-3'. The thermocycling conditions for qPCR were as follows: Initial denaturation for $3 \mathrm{~min}$ at $95^{\circ} \mathrm{C}$, followed by 45 cycles of $95^{\circ} \mathrm{C}$ for $10 \mathrm{sec}$ and $58^{\circ} \mathrm{C}$ for $45 \mathrm{sec}$. Data were acquired at the end of the annealing/extension phase. Melt curve analysis was performed at the end of each run from 58 to $95^{\circ} \mathrm{C}$.

Western blot analysis. Cells were lysed in radioimmunoprecipitation assay buffer (Pierce; Thermo Fisher Scientific, Inc.), and protein was quantified using Coomassie Blue protein standards (Pierce; Thermo Fisher Scientific, Inc.). Protein samples (30 'g) were subjected to SDS-PAGE (10\% gel) and transferred onto polyvinylidene difluoride membranes (EMD Millipore, Billerica, MA, USA). The membranes were incubated with blocking buffer [5\% skimmed milk in Tris-buffered saline with $0.1 \%$ Tween-20 (TBS-T)] at room temperature for $1 \mathrm{~h}$ and then proteins were detected with the following antibodies at 1:500 dilution, incubated overnight at $4^{\circ} \mathrm{C}$ : Anti-cluster of differentiation (CD)63 antibody (cat. no. 25682-1-AP; ProteinTech Group, Inc., Chicago, IL, USA), anti-CD81 antibody (cat. no. ab109201; Abcam, Cambridge, MA, USA), anti-cytochrome c oxidase IV (Cox IV; cat. no. sc58348, Santa Cruz Biotechnology, Inc., Dallas, TX, USA), anti-B-cell lymphoma 2 (Bcl-2; cat no. 2870; Cell Signaling Technology, Inc., Danvers, MA, USA), anti-B-cell lymphoma extra-large (Bcl-xl; cat no. 2764; Cell Signaling Technology, Inc.), anti-myeloid cell leukemia-1 (Mcl-1; cat no. 94296; Cell Signaling Technology, Inc.) and anti- $\beta$-actin antibody (cat no. ab8227; Abcam). The membranes were washed with TBS-T, then incubated with horseradish peroxidase-conjugated anti-rabbit or anti-mouse secondary antibody (cat. nos. AP182P and AP308P, respectively; 1:10,000 dilution; Sigma-Aldrich; Merck KGaA, Darmstadt, Germany) at room temperature for $2 \mathrm{~h}$. Detection was performed using western blot detection reagents (Odyssey; LI-COR Biosciences, Lincoln, NE, USA).

Lentiviral vector-mediated gene KD or OE. The AHIF-KD target sequence was: 5'-GATCCAAAGCTCTGAGTAA-3'. The AHIF-OE sequence (NCBI accession no. NR_045406.1) was constructed by Hanyin Ltd., Co. (Shanghai, China). The recombinant lentivirus and negative control (NC; PHY-008 for AHIF-OE NC and PHY-310 for AHIF-KD NC) lentivirus were prepared and titered to 109 transfection units/ml (Hanyin Ltd., Co.). After 48 h, the efficiency of AHIF-KD or AHIF-OE was confirmed using RT-qPCR as aforementioned. To obtain stably transfected cells, GBM cells (U87-MG, U251-MG, A172 and T98G) with AHIF-KD or AHIF-OE cells and respective control cells were seeded in 6-well dishes at a density of 1x105 cells/well. The cells were then infected with the same virus titer on the following day with $8 \mu \mathrm{g} / \mathrm{ml}$ Polybrene (Maokang Co., Shanghai, China). At $72 \mathrm{~h}$ after viral infection, the culture medium was replaced with 

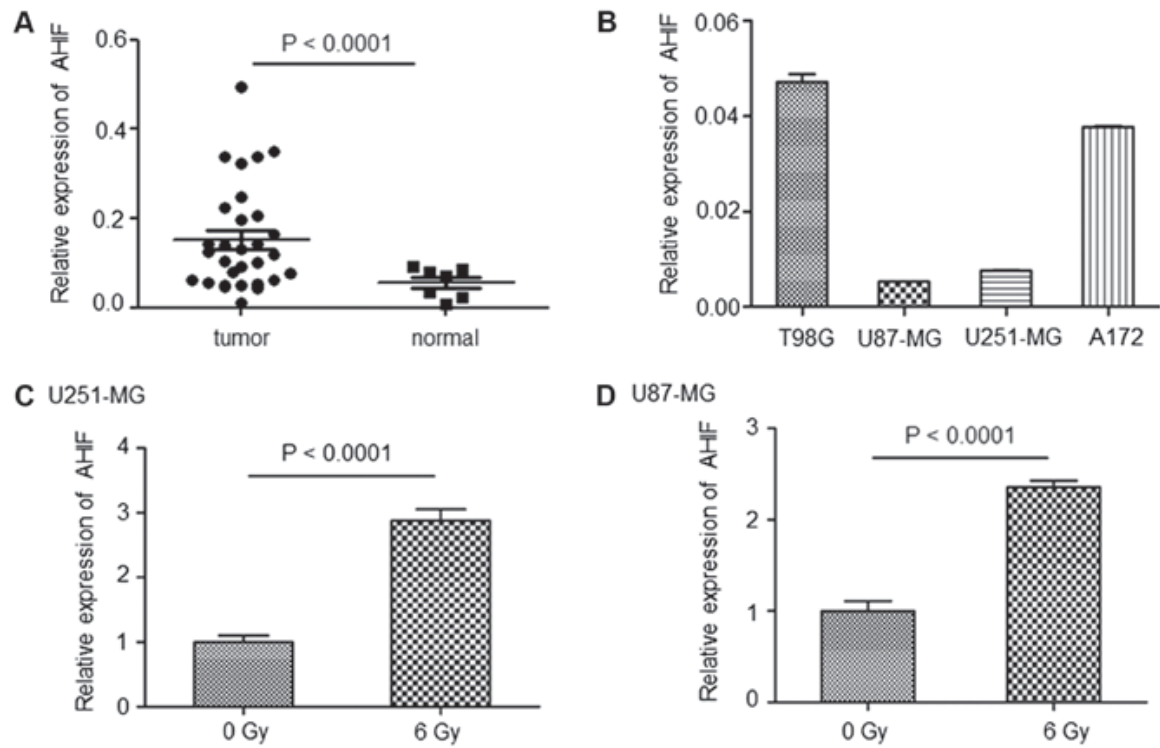

Figure 1. AHIF is highly expressed in GBM and in response to radiotherapy. (A) Expression of AHIF in normal tissues (n=7) and GBM tissues (n=28). (B) Relative AHIF mRNA levels in GBM cells, including U87-MG, U251-MG, A172 and T98G cell lines. (C) Relative AHIF mRNA levels in U251-MG cells treated with 6 or 0 Gy radiation. (D) Relative AHIF mRNA levels in U87-MG cells treated with 6 or 0 Gy radiation. AHIF, antisense transcript of hypoxia-inducible factor $1 \alpha$; GBM, glioblastoma multiforme.

Dulbecco's modified Eagle's medium (DMEM; Hyclone; GE Healthcare) containing $4 \mu \mathrm{g} / \mathrm{ml}$ puromycin supplemented with $10 \%$ FBS. The puromycin-resistant cells were amplified in medium containing $2 \mu \mathrm{g} / \mathrm{ml}$ puromycin for 7 days and then transferred to a medium without puromycin.

Cell Counting Kit-8 (CCK-8) assay. A CCK-8 assay (Dojindo Molecular Technologies, Inc., Kumamoto, Japan) was performed according to the manufacturer's protocol. In brief, exosome-treated GBM cells (U87-MG, U251-MG, A172 and T98G), with AHIF-KD or AHIF-OE cells and respective control cells, were cultured at equal cell density (2,500 cells/100 $\mu$ l per well) in 96-well plates for continuous detection over a 5 -day period. The culture was terminated by adding $10 \mu$ l CCK-8 $(5 \mathrm{mg} / \mathrm{ml})$ to the culture medium. After $2 \mathrm{~h}$, the wells were analyzed using a microplate reader (BioTek Elx800; BioTek Instruments, Inc., Winooski, VT, USA) at $490 \mathrm{~nm}$.

Invasion assay. GBM cells (U87-MG, U251-MG, A172 and T98G), with AHIF-KD or AHIF-OE cells and respective control cells, at $1 \times 10^{4}$ cells $/ 100 \mu 1$ were plated in the upper chambers of Matrigel-coated Transwell assay inserts (EMD Millipore) in $200 \mathrm{ml}$ serum-free DMEM. The inserts were then placed into wells of a 24-well plate containing DMEM with $10 \% \mathrm{FBS}$ as a chemoattractant. After $24 \mathrm{~h}$ at $37^{\circ} \mathrm{C}$, the top layer of the insert was wiped with a cotton swab to remove remaining cells. The invading cells on the lower surface were stained with $0.1 \%$ crystal violet at room temperature for $1 \mathrm{~h}$ and images were captured using digital microscopy. The number of cells in five random fields of each chamber was determined, and the mean number of cells was calculated.

Cell apoptosis analysis. Apoptosis was analyzed by translocation of phosphatidylserine to the cell surface using an Annexin and DAPI Apoptosis Detection kit (BD Biosciences,
Franklin Lakes, NJ, USA). GBM cells (U87-MG, U251-MG, A172 and T98G), with AHIF-KD or AHIF-OE cells and respective control cells, were treated with 6-Gy radiation, then collected and washed in ice-cold PBS. Cells were stained with AnnexinV-fluorescein isothiocyanate and DAPI for $30 \mathrm{~min}$ in the dark. Cell apoptosis was analyzed using BD CellQuest ${ }^{\mathrm{TM}}$ Pro software (BD Biosciences) on a FACSAria flow cytometer (BD Biosciences). Fluorescence was captured with emission wavelength of $488 \mathrm{~nm}$.

Exosome isolation and co-culture. In order to isolate exosomes, GBM cells (U87-MG, U251-MG, A172 and T98G), with AHIF-KD or AHIF-OE cells and respective control cells, were cultured for $48 \mathrm{~h}$ and the supernatant was collected. The supernatants were then centrifuged twice $(1,000 \mathrm{x} \mathrm{g}$ for $10 \mathrm{~min}$ and $3,000 \mathrm{xg}$ for $30 \mathrm{~min}$ at $4^{\circ} \mathrm{C}$ ) to deplete them of the cells and fragments. Then, Total Exosome Isolation Reagent (Thermo Fisher Scientific, Inc.) was added overnight, followed by centrifugation $10,000 \mathrm{xg}$ for $1 \mathrm{~h}$ at $4^{\circ} \mathrm{C}$. Exosomes were resuspended in PBS and stored at $-80^{\circ} \mathrm{C}$. The concentration of exosomes was determined using a Bicinchoninic Acid Protein assay. Exosomes were then added to $10^{5} \mathrm{GBM}$ cells at a concentration of $50 \mathrm{ng} / \mathrm{ml}$ serum-free DMEM for $24 \mathrm{~h}$. AHIF-OE cells were treated with exosomal inhibitor GW4869 $(10 \mu \mathrm{M}$ for $24 \mathrm{~h}$ at $37^{\circ} \mathrm{C}$ with $5 \% \mathrm{CO}_{2}$ ) prior to collection of the supernatant.

Electron microscopic observation of exosomes. The exosome suspension was added to an equal volume of $4 \%$ paraformaldehyde (Nacalai Tesque, Inc., Kyoto, Japan), and the mixture was applied to a Formvar/carbon film-coated transmission electron microscope (TEM) grid (Alliance Biosystems, Inc., Osaka, Japan). Subsequently, the sample was fixed by incubation with $1 \%$ glutaraldehyde for $5 \mathrm{~min}$, washed with PBS, and incubated with $1 \%$ uranyl acetate for $5 \mathrm{~min}$. The sample was observed under a TEM (Hitachi H-7650; Hitachi, Ltd., Tokyo, Japan). 

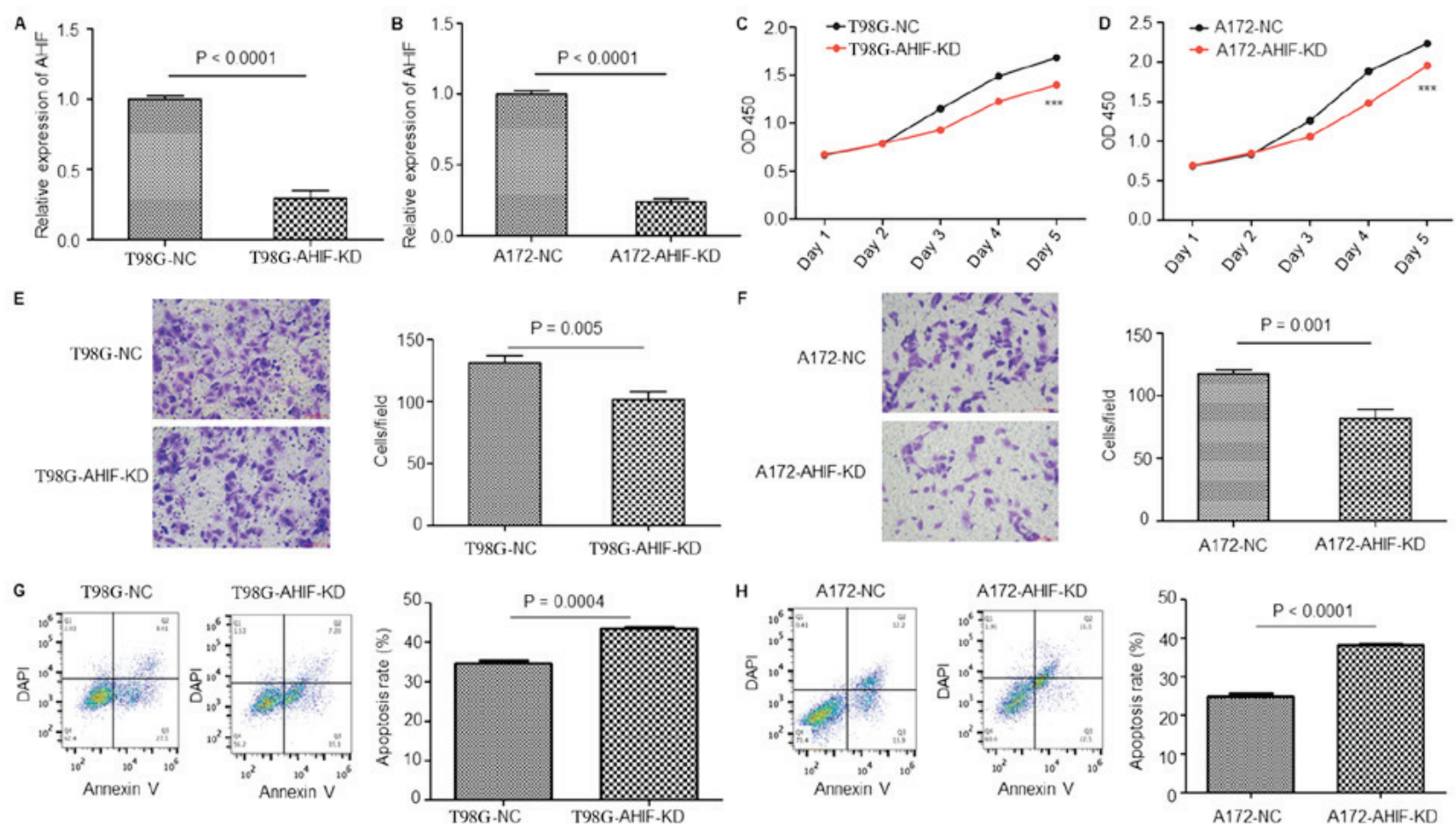

Figure 2. Suppression of AHIF in GBM cells decreases viability, invasion and radioresistance. Relative AHIF levels in (A) T98G and (B) A172 cells with or without AHIF KD. Cell Counting Kit-8 analysis of viability in (C) T98G-AHIF-KD and control cells, and (D) T98G-AHIF-KD and control cells. Invasion analysis of (E) T98G-AHIF-KD and control cells, and (F) T98G-AHIF-KD and control cells. Apoptosis analysis of (G) T98G-AHIF-KD and control cells, and (H) T98G-AHIF-KD and control cells. All experiments were performed three times. AHIF, antisense transcript of hypoxia-inducible factor 1 $\alpha$; GBM, glioblastoma multiforme; KD, knockdown; NC, negative control; OD, optical density.

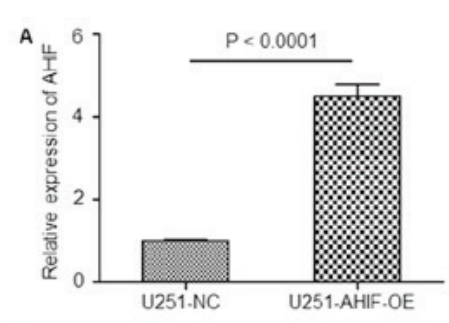

E

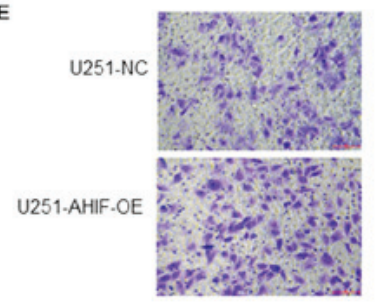

G

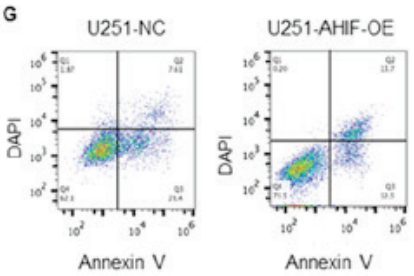

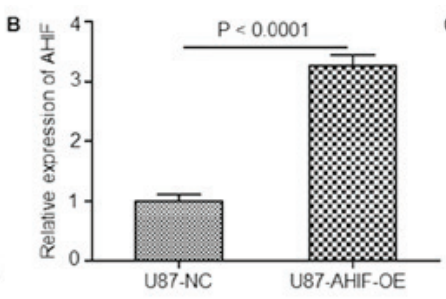
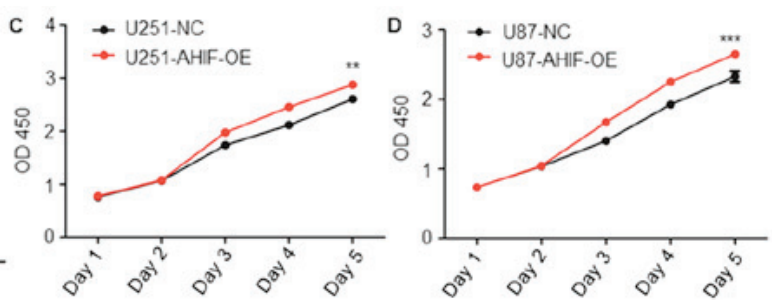

F
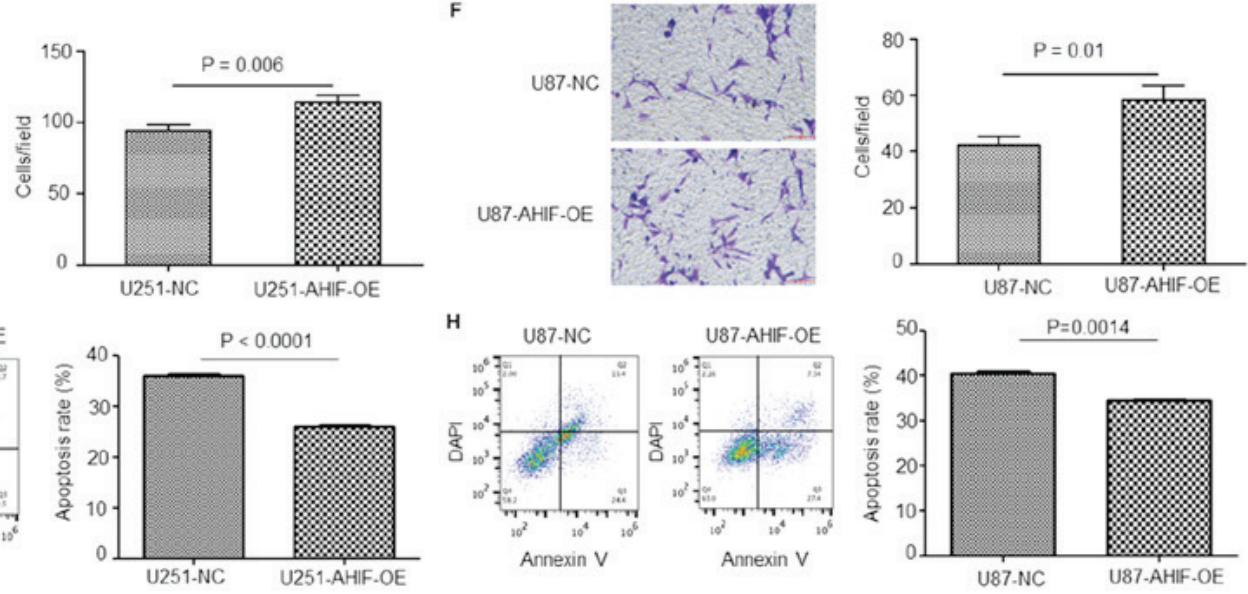

Figure 3. Overexpression of AHIF in GBM cells enhances viability, invasion and radioresistance. Relative AHIF levels in (A) U251-MG and (B) U87-MG cells with or without AHIF OE. Cell Counting Kit-8 analysis of viability in (C) U251-AHIF-OE and control cells, and (D) U87-AHIF-OE and control cells. Invasion analysis of (E) U251-AHIF-OE and control cells, and (F) U87-AHIF-OE and control cells. Apoptosis analysis of (G) U251-AHIF-OE and control cells, and (H) U87-AHIF-OE and control cells. All experiments were performed three times. AHIF, antisense transcript of hypoxia-inducible factor 1 $\alpha$; GBM, glioblastoma multiforme; OE, overexpression; NC, negative control; OD, optical density.

Exosome labeling with PKH67. Exosomes derived from AHIF-KD or AHIF-OE cells were labeled with PKH67, a Green
Fluorescent Labeling kit (Sigma-Aldrich; Merck KGaA). The concentration of PKH67 used for exosome labeling was $2 \mu \mathrm{M}$ 

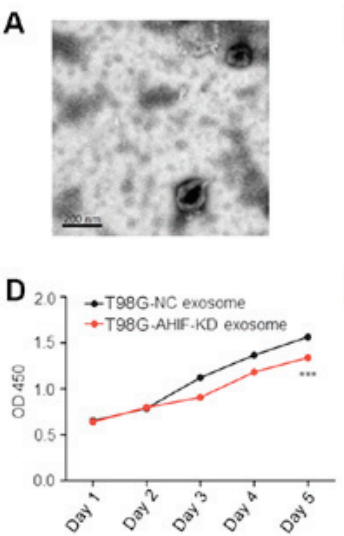

G
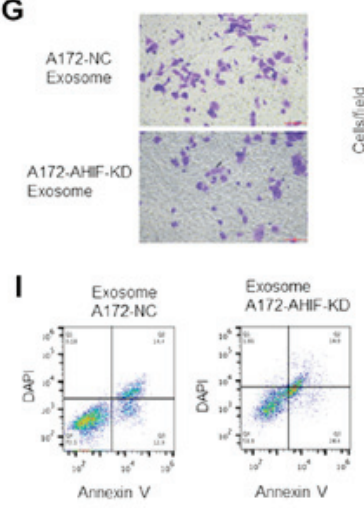

B
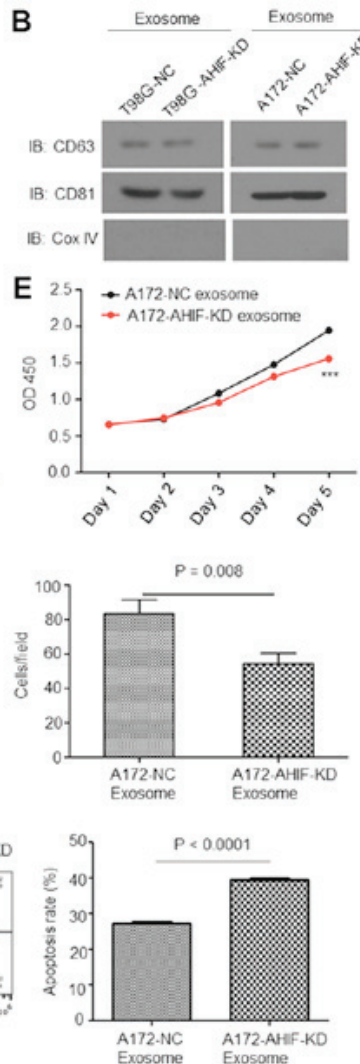

C
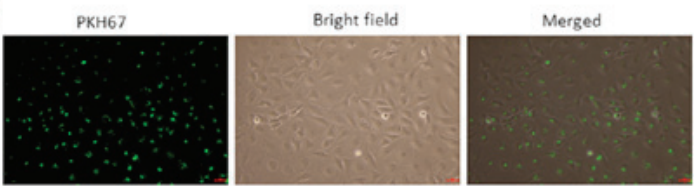

F
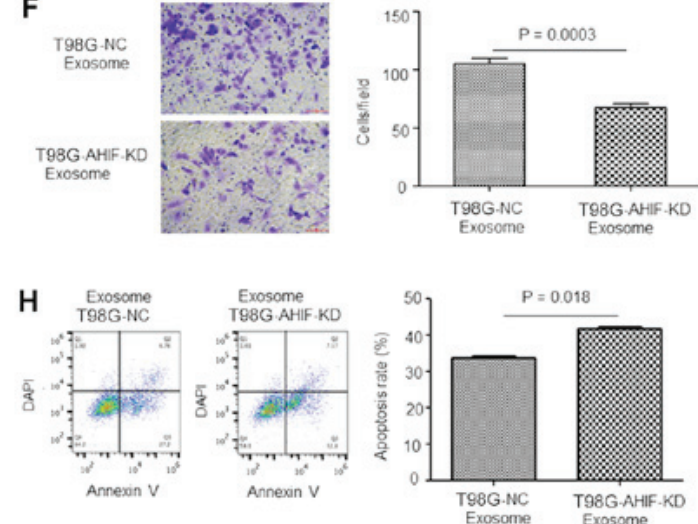

Figure 4. Exosomes derived from AHIF-KD GBM cells inhibit cell viability, invasion and radioresistance. (A) Representative electron micrograph of exosomes isolated from GBM cells. (B) Western blot analysis indicating the presence of CD81 and CD63 in isolated exosomes. (C) GBM cells endocytose exosomes. Fluorescence microscopy results of exosomes co-cultured with A172 cells. Exosomes were stained with PHK67 (green). Cell Counting Kit-8 analysis of viability in (D) T98G and (E) A172 cells treated with exosomes derived from AHIF-KD or control cells. Invasion analysis of (F) T98G and (G) A172 cells treated with exosomes derived from AHIF-KD or control cells. Apoptosis analysis of (H) T98G and (I) A172 cells treated with exosomes derived from AHIF-KD or control cells. All experiments were performed three times. AHIF, antisense transcript of hypoxia-inducible factor 1 $\alpha$; GBM, glioblastoma multiforme; KD, knockdown; $\mathrm{CD}$, cluster of differentiation; OD, optical density; NC, negative control; IB, immunoblot.

per exosome from $5 \times 10^{5}$ cells. The labeled exosomes were assessed using an inverted fluorescence microscopy (Olympus CKX41; Olympus Corporation, Tokyo, Japan).

Statistical analysis. Results are presented as the mean \pm standard error of the mean. One-way analysis of variance with Tukey's test was conducted to compare multiple groups. All statistical analyses were performed using SPSS for Windows (version 17.0; SPSS, Inc., Chicago, IL, USA). Two-tailed P<0.05 was considered to indicate a statistically significant difference.

\section{Results}

AHIF is highly expressed in GBM and in response to radiotherapy. Fresh tissues were collected from patients with GBM as well as adjacent non-cancerous tissues. The results of RT-qPCR indicated that AHIF expression was significantly upregulated in GBM tissues compared with in normal tissues (Fig. 1A). AHIF expression was then investigated in an array of GBM cell lines (U87-MG, U251-MG, A172 and T98G). As indicated in Fig. 1B, T98G and A712 cells exhibited increased levels of AHIF expression compared with in U87-MG and U251-MG cells. Furthermore, AHIF expression was increased in U87-MG and U251-MG cells following irradiation (Fig. 1C and D), indicating that AHIF expression may be affected by radiotherapy.
Suppression of AHIF in GBM cells decreases cell viability, invasion and radioresistance. To investigate the function of AHIF in GBM progression and radiotherapy, T98G and A172 cells with stable KD of AHIF were constructed using a lentivirus. As presented in Fig. 2A and B, AHIF was effectively inhibited in AHIF-KD cells compared with in the NC cells. CCK-8 analysis of these cells suggested that the viability of AHIF-KD cells was significantly decreased compared with that of NC cells (Fig. 2C and D). Invasion assay results demonstrated that AHIF-KD cells had a significantly decreased invasive capacity compared with that of NC cells (Fig. 2E and F). Furthermore, an apoptosis assay of AHIF-KD cells following 6-Gy treatment indicated that the proportion of apoptotic cells was significantly increased (Fig. 2G and H). In summary, these results indicate that KD of AHIF in GBM cells decreased the viability and invasive ability, and increased the proportion of apoptotic cells following radiotherapy.

OE of AHIF in GBM cells increases cell viability, invasion and radioresistance. To further clarify the function of AHIF in GBM progression and radiotherapy, GBM cells with stable $\mathrm{OE}$ of AHIF were constructed using a lentivirus. As presented in Fig. 3A and B, AHIF was effectively overexpressed in AHIF-OE U87-MG and U251-MG cells compared with in the NC cells. CCK- 8 analysis of these cells identified that the viability of AHIF-OE cells was significantly increased 

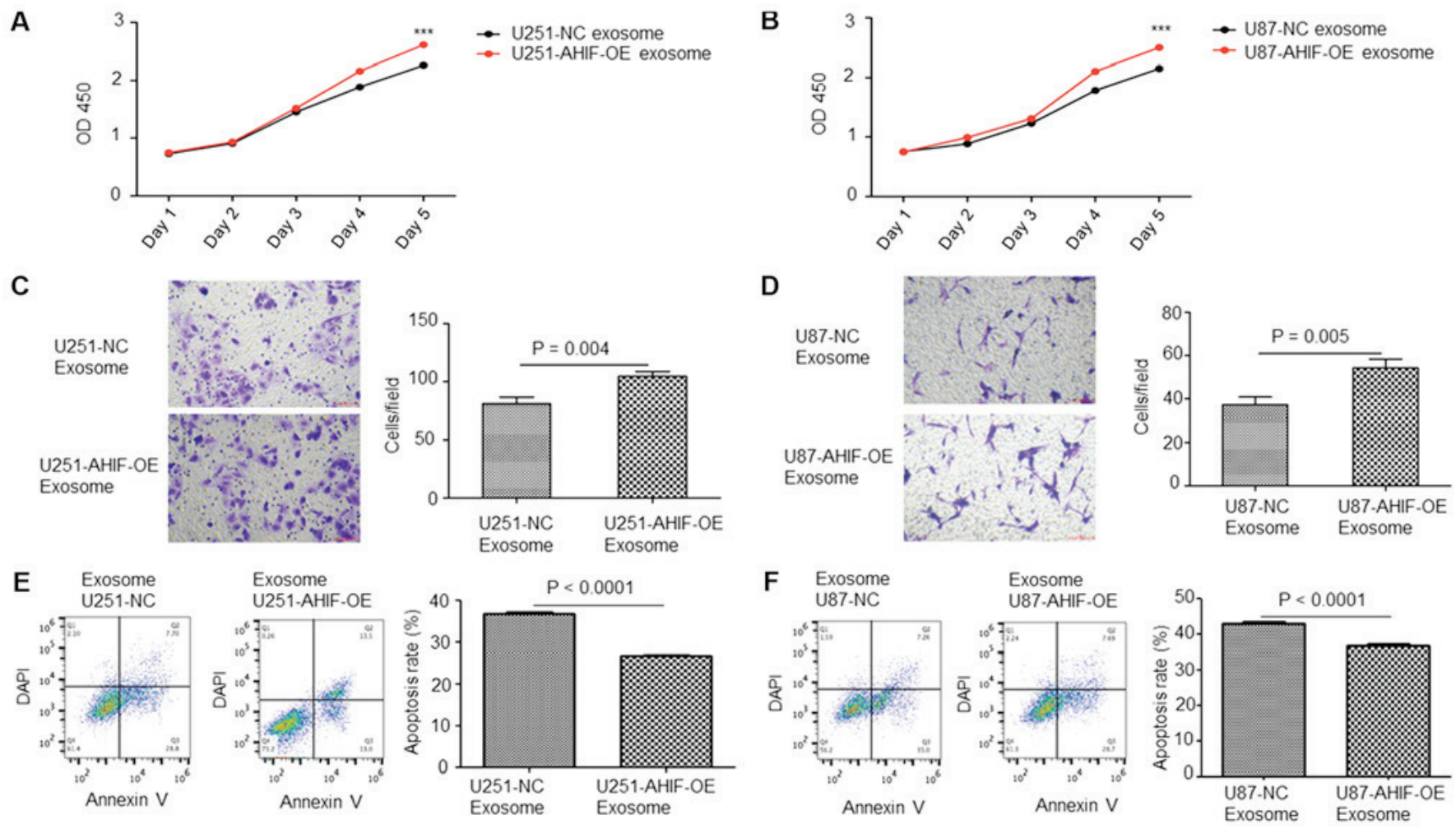

Figure 5. Exosomes derived from AHIF-OE GBM cells promote cell viability, invasion and radioresistance. Cell Counting Kit-8 analysis of viability of (A) U251-MG and (B) U87-MG cells treated with exosomes derived from AHIF-OE or control cells. Invasion analysis of (C) U251-MG and (D) U87-MG cells treated with exosomes derived from AHIF-OE or control cells. Apoptosis analysis of (E) U251-MG and (F) U87-MG cells treated with exosomes derived from AHIF-OE or control cells. All experiments were performed three times. AHIF, antisense transcript of hypoxia-inducible factor 1 $\alpha$; OE, overexpression; OD, optical density; NC, negative control.

compared with that of NC cells (Fig. 3C and D). Invasion assay results demonstrated that OE of AHIF significantly increased the cell invasive ability (Fig. 3E and F). Furthermore, an apoptosis assay of AHIF-OE cells following 6-Gy treatment indicated that the proportion of apoptotic cells was significantly decreased (Fig. 3G and H). In summary, these results indicate that OE of AHIF in GBM cells increased the viability and invasive ability, and decreased the proportion of apoptotic cells following radiotherapy.

Exosomes derived from AHIF-KD cells inhibit GBM cell viability, invasion and radioresistance. Exosomes have previously been identified to serve important functions in hypoxia and tumor progression $(26,27)$. Therefore, in the present study, it was investigated whether AHIF was able to regulate GBM progression and radiotherapy via exosomes. Exosomes were isolated from GBM cell medium and their morphology was observed under a TEM (Fig. 4A). Western blot analysis indicated that the exosomes were enriched with the exosomal markers CD63 and CD81, but not the mitochondrial marker Cox IV (Fig. 4B), indicating that exosomes had been successfully isolated. Fig. 4C presents fluorescence microscopy images of GBM cells co-cultured with exosomes [stained with PKH67 (green)]. CCK-8 analysis indicated that the viability of cells co-cultured with exosomes from AHIF-KD cells was significantly decreased compared with cells treated with exosomes derived from control cells (Fig. 4D and E). Invasion assay results indicated that cells co-cultured with exosomes derived from AHIF-KD cells exhibited significantly decreased invasive ability compared with cells treated with exosomes derived from control cells (Fig. 4F and G). Furthermore, an apoptosis assay of these cells following 6-Gy treatment indicated that the group co-cultured with exosomes derived from AHIF-KD cells contained a significantly increased proportion of apoptotic cells compared with the cell group treated with exosomes derived from control cells (Fig. 4H and I). In summary, these results indicate that AHIF-KD cells inhibited GBM cell viability, invasion and radioresistance via exosomes.

Exosomes derived from AHIF-OE cells promote cell viability, invasion and radioresistance. Exosomes were collected from AHIF-OE cells. CCK-8 analysis indicated that the viability of cells co-cultured with exosomes derived from AHIF-OE cells was significantly increased compared with cells treated with exosomes derived from control cells (Fig. 5A and B). Invasion assay results suggested that cells co-cultured with exosomes derived from AHIF-OE cells exhibited significantly increased invasive ability compared with cells treated with exosomes derived from control cells (Fig. 5C and D). Furthermore, an apoptosis assay of these cells following 6-Gy treatment indicated that the cell group co-cultured with exosomes derived from AHIF-OE cells contained a significantly decreased percentage of apoptotic cells compared with the cell group treated with exosomes derived from control cells (Fig. 5E and F). In summary, these results indicate that AHIF-OE GBM cells promoted viability, invasion and radioresistance via exosomes. 

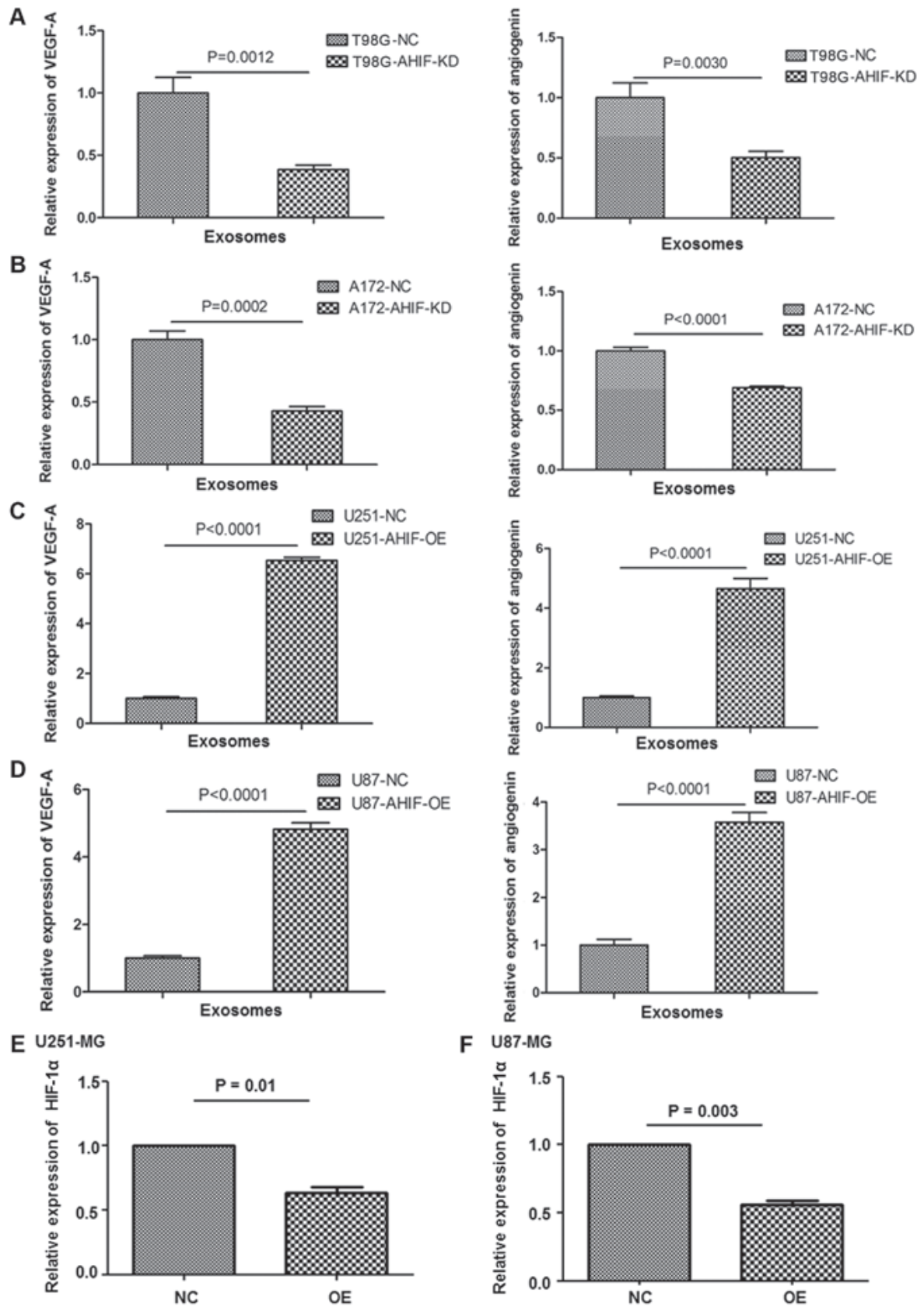

Figure 6. AHIF regulates factors associated with migration and angiogenesis in exosomes. Relative exosomal VEGF and angiogenin levels in (A) T98G cells and (B) A172 cells with or without AHIF knockdown. Relative exosomal VEGF and angiogenin levels in (C) U251-MG cells and (D) U87-MG cells with or without AHIF overexpression. Relative HIF-1 $\alpha$ levels in (E) U251-MG cells and (F) U87-MG cells with or without AHIF overexpression. All experiments were performed three times. AHIF, antisense transcript of hypoxia-inducible factor $1 \alpha$; VEGF, vascular endothelial growth factor, HIF-1 $\alpha$, hypoxia inducible factor $1 \alpha$; NC, negative control; OE, overexpression; KD, knockdown.

AHIF regulates factors associated with invasion and apoptosis. In order to clarify the underlying molecular mechanisms of AHIF in GBM progression and radiotherapy, invasion and angiogenic genes were analyzed in AHIF-KD and AHIF-OE cells. Expression levels of exosomal VEGF and angiogenin were significantly decreased following KD of AHIF (Fig. 6A and B). By contrast, the expression levels of exosomal VEGF and angiogenin were significantly increased following $\mathrm{OE}$ of AHIF (Fig. 6C and D). Significantly downregulated HIF-1 $\alpha$ expression was observed in AHIF-OE cells (Fig. 6E and F).

Furthermore, the expression levels of anti-apoptotic Bcl-2, Bcl-xl and Mcl-1 were analyzed in AHIF-KD, AHIF-OE and control cells. The western blot results indicated that the expression of anti-apoptotic Bcl-2, Bcl-xl and Mcl-1 was decreased in AHIF-KD cells (Fig. 7A). By contrast, the levels of these proteins were increased in AHIF-OE cells (Fig. 7B). These data indicated that AHIF regulates factors associated with invasion and apoptosis.

Suppression of exosome secretion in AHIF-OE GBM cells decreases the invasive and anti-apoptosis abilities of GBM cells. To further verify the function of exosomes in the effect of AHIF expression on GBM cells, exosome generation was blocked with the exosomal release inhibitor GW4869 $(31,32)$. As presented in Fig. 8A, exosome release was effectively suppressed following GW4869 treatment. Invasion assay results indicated 

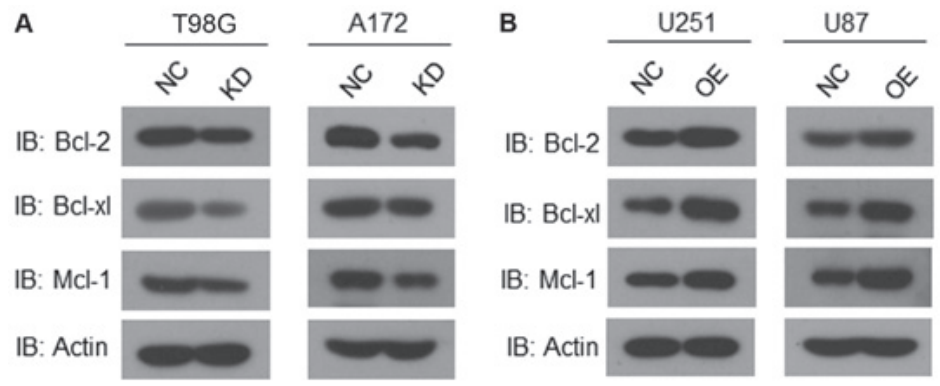

Figure 7. AHIF regulates factors associated with apoptosis. (A) Western blot analysis of Bcl-2, Bcl-xl, Mcl-1 and actin in T98G cells and A172 cells with or without AHIF knockdown. (B) Western blot analysis of Bcl-2, Bcl-xl, Mcl-1 and actin in U251-MG cells and U87-MG cells with or without AHIF overexpression. All experiments were performed three times. AHIF, antisense transcript of hypoxia-inducible factor 1 $\alpha$; Bcl-2, B-cell lymphoma 2; Bcl-xl, B-cell lymphoma extra-large; Mcl-1, myeloid cell leukemia-1; NC, negative control; KD, knockdown; OE overexpression; IB, immunoblot.

A

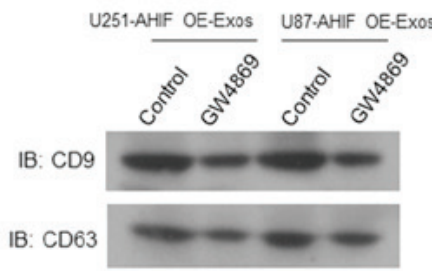

B U251-AHIF OEExos

Control
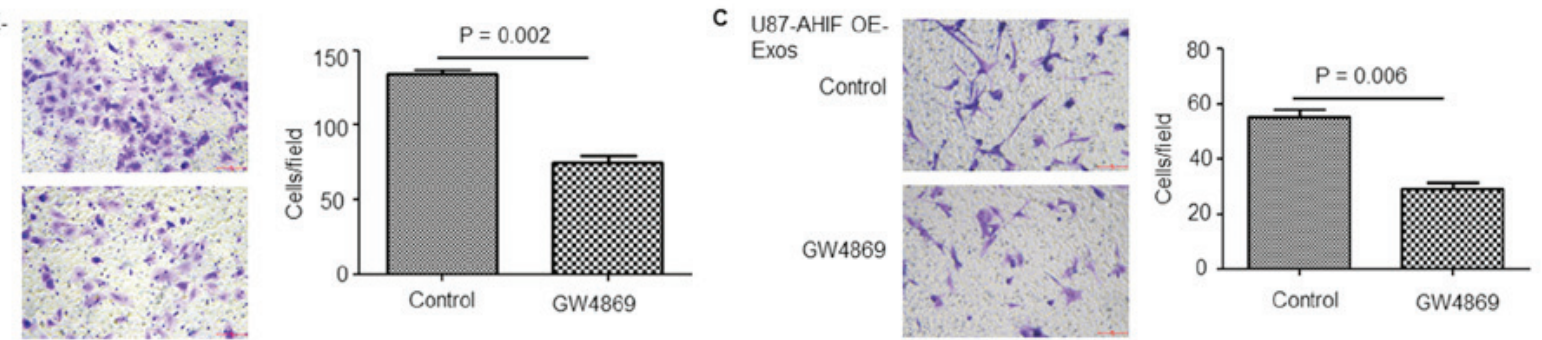

D

U251-AHIF OE-Exos
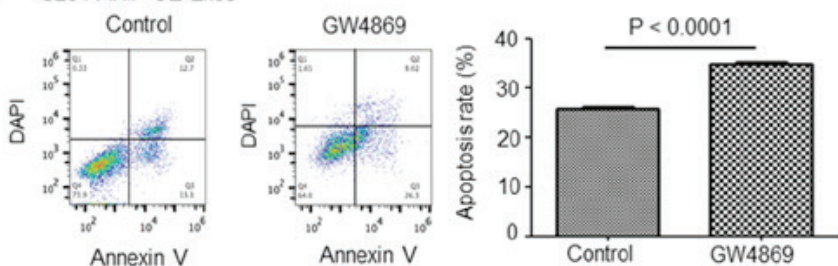

E U87-AHIF OE-EXOS
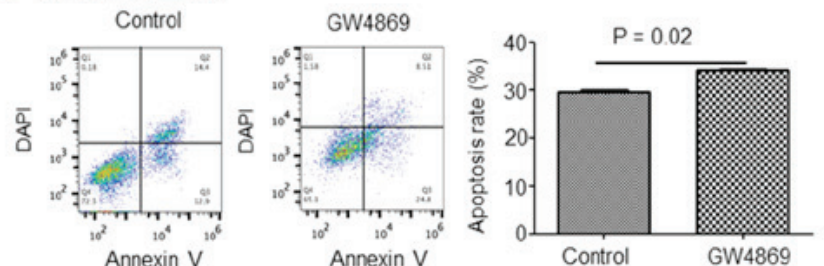

Figure 8. Suppression of exosome secretion in AHIF-OE GBM cells decreases the invasive and anti-apoptosis abilities of GBM cells. (A) Western blot analysis of exosomal markers CD9 and CD63, indicating that GW4869 treatment effectively decreased exosome release. Invasion analysis of (B) U251-MG and (C) U87-MG cells treated with exosomes derived from AHIF-OE cells, with or without GW4869. Apoptosis analysis of (D) U251-MG and (E) U87-MG cells treated with exosomes derived from AHIF-OE cells with or without GW4869. All experiments were performed three times. AHIF, antisense transcript of hypoxia-inducible factor $1 \alpha$; GBM, glioblastoma multiforme; OE, overexpression; $\mathrm{CD}$, cluster of differentiation; Exos, exosomes; IB, immunoblot.

that GW4869 significantly decreased GBM cell invasion induced by exosomes derived from AHIF-OE cells (Fig. 8B and C). Furthermore, the apoptosis assay indicated that GW4869 treatment significantly inhibited the cell survival ability induced by exosomes derived from AHIF-OE cells (Fig. 8D and E). These results provided further evidence that AHIF promotes the invasive and anti-apoptosis abilities of GBM cells via exosomes.

\section{Discussion}

Despite the availability of aggressive therapeutic regimens, the majority of patients with GBM suffer recurrence due to its molecular heterogeneity (33-35). Consequently, a number of geneticfactors associated with GBM progressionandradiotherapy have been investigated, including isocitrate dehydrogenase mutations, $1 \mathrm{p} 19 \mathrm{q}$ deletion, $\mathrm{O}^{6}$-methylguanine-DNA methyltransferase promoter methylation and epidermal growth factor receptor variant III amplification (36-39). In the present study, it was identified that AHIF was significantly upregulated in cancerous GBM tissues as well as radioresistant GBM cells, indicating that AHIF may be a novel biomarker for GBM progression and radioresistance. A non-cancerous glial cell line was not included in the present study, which may need further clarification. These results were consistent with a previous study, which identified that AHIF is upregulated in breast cancer tissues (18). However, the function and underlying molecular mechanisms of AHIF are largely unknown. In the present study, the function of AHIF in GBM cells was revealed through KD or OE of AHIF. The results indicated that AHIF regulates cell viability, invasion and apoptosis in response to radiotherapy, 
which may provide a therapeutic target. Although the differences between groups were relatively small in the CCK-8 assay, these were still observed to be significant. However, the difference between groups was more obvious in the invasion and apoptosis experiments, which may be due to regulatory effects of the tumor microenvironment on tumor cells. LncRNAs such as HOTAIR have been observed to be dysregulated in GBM and required for GBM cell proliferation (40).

The results of the present study raise the question of how AHIF promotes tumor invasiveness and radioresistance. The expression of HIFla is negatively regulated by AHIF, which forms a double-stranded RNA molecule with the antisense transcript of HIF-1 $\alpha(15,16)$. Consistent with this, downregulated HIF-1 $\alpha$ expression was observed in AHIF-OE cells. HIF-1 $\alpha$ stabilizes the tumor suppressor gene p53 (41). Inhibition of HIF-1 $\alpha$ by AHIF during sustained hypoxia results in the loss of p53 and subsequent tumor cell proliferation (41). The results of the present study indicated that the expression of anti-apoptotic Bcl-2, Bcl-xl and $\mathrm{Mcl}-1$ decreased in AHIF-KD cells; by contrast, these proteins were increased in AHIF-OE cells. Thus, AHIF-mediated p53 downregulation and anti-apoptosis may be one of the mechanisms by which AHIF conveys more aggressive tumor behavior and radioresistance. Furthermore, AHIF-regulated exosomal secretion of VEGF and angiogenin may a novel mechanism responsible for invasion and radioresistance.

Exosomes are nanovesicles released by tumor cells to modulate tumor progression. Accumulating evidence has revealed that glioblastoma-derived exosomes contain multiple pro-angiogenic factors that induce proliferation and progression (42-44). VEGF-A has been identified to be overexpressed in hypoxic GBM-derived exosomes (45). Considering the classic function of hypoxia in angiogenesis and invasion, angiogenic genes in exosomes derived from AHIF-KD and OE cells were analyzed. In the present study, it was observed that AHIF KD or OE in GBM cells altered the content of VEGF-A and angiogenin in secreted exosomes, indicating that AHIF promotes glioblastoma progression and radioresistance via exosomes. In addition, exosomes collected from GBM cells with AHIF KD or OE altered the viability, invasion and apoptosis in response to radiotherapy of GBM cells. Although the interaction between AHIF and HIF-1 $\alpha$ has been suggested in a previous study (19), the molecular mechanisms underlying the regulation of AHIF, VEGF and angiogenin require further investigation. Increased AHIF expression has been observed to be in parallel with that of VEGF (20). Furthermore, hypoxic glioblastoma releases exosomal VEGF to induce permeability of the blood-brain barrier (46).

To the best of our knowledge, the present study is the first to establish that AHIF promotes glioblastoma progression and radioresistance via exosomes. This could serve as a potential therapeutic target in the treatment of GBM.

\section{Acknowledgements}

Not applicable.

\section{Funding}

The present study was supported by the National Science Foundation of Fujian Province (grant nos. 2017J0105 and 2018J01210) and the National Science Foundation of China (grant nos. 81671203 and 81874215).

\section{Availability of data and materials}

All data generated or analyzed during this study are included in this published article.

\section{Authors' contributions}

$\mathrm{XD}, \mathrm{KL}, \mathrm{YQ}$ and RL contributed to the experimental design, performing experiments, acquiring data, analyzing data, providing reagents and writing the manuscript. ZZhuang, BC, ZZhou, SZ, GL, FZ, YL, YM, ZL and RH contributed to performing experiments and acquiring data.

\section{Ethics approval and consent to participate}

The present study was approved by the Ethics Committee of Renji Hospital, School of Medicine, Shanghai Jiao Tong University (Shanghai, China). Written informed consent was obtained from patients for participation in the study.

\section{Patient consent for publication}

Glioma tissues were collected from patients at Shanghai Renji Hospital, School of Medicine, Shanghai Jiao Tong University, following acquisition of written informed consent for publication from the patients and with institutional review board approval of the hospital. All patients obtained a confirmed diagnosis of glioblastoma following resection.

\section{Competing interests}

The authors declare that they have no competing interests.

\section{References}

1. Ostrom QT, Gittleman H, Farah P, Ondracek A, Chen Y, Wolinsky Y, Stroup NE, Kruchko C and Barnholtz-Sloan JS: CBTRUS statistical report: Primary brain and central nervous system tumors diagnosed in the United States in 2006-2010. Neuro Oncol 15 (Suppl 2): ii1-ii56, 2013.

2. Cuddapah VA, Robel S, Watkins S and Sontheimer H: A neurocentric perspective on glioma invasion. Nat Rev Neurosci 15: 455-465, 2014.

3. Suvà ML, Rheinbay E, Gillespie SM, Patel AP, Wakimoto H, Rabkin SD, Riggi N, Chi AS, Cahill DP, Nahed BV, et al: Reconstructing and reprogramming the tumor-propagating potential of glioblastoma stem-like cells. Cell 157: 580-594, 2014.

4. Caruso C, Carcaterra M and Donato V: Role of radiotherapy for high grade gliomas management. J Neurosurg Sci 57: 163-169, 2013.

5. Debus C, Waltenberger M, Floca R, Afshar-Oromieh A, Bougatf N, Adeberg S, Heiland S, Bendszus M, Wick W, Rieken $\mathrm{S}$, et al: Impact of 18F-FET PET on target volume definition and tumor progression of recurrent high grade glioma treated with carbon-ion radiotherapy. Sci Rep 8: 7201, 2018.

6. Yadav VN, Altshuler D, Kadiyala P, Zamler D, Comba A, Appelman H, Dunn P, Koschmann C, Castro MG and Löwenstein PR: Molecular ablation of tumor blood vessels inhibits therapeutic effects of radiation and bevacizumab. Neuro Oncol 20: 1356-1367, 2018.

7. Song X, Shao Y, Jiang T, Ding Y, Xu B, Zheng X, Wang Q, Chen X, $\mathrm{Gu} \mathrm{W}, \mathrm{Wu} \mathrm{C}$, et al: Radiotherapy upregulates programmed death ligand-1 through the pathways downstream of epidermal growth factor receptor in glioma. EBioMedicine 28: 105-113, 2018.

8. Ørom UA and Shiekhattar R: Long noncoding RNAs usher in a new era in the biology of enhancers. Cell 154: 1190-1193, 2013. 
9. Wapinski $\mathrm{O}$ and Chang HY: Long noncoding RNAs and human disease. Trends Cell Biol 21: 354-361, 2011.

10. Zhang JX, Han L, Bao ZS, Wang YY, Chen LY, Yan W, Yu SZ, $\mathrm{Pu}$ PY, Liu N, You YP, et al; Chinese Glioma Cooperative Group: HOTAIR, a cell cycle-associated long noncoding RNA and a strong predictor of survival, is preferentially expressed in classical and mesenchymal glioma. Neuro Oncol 15: 1595-1603, 2013.

11. Li Z, Xu C, Ding B, Gao M, Wei X and Ji N: Long non-coding RNA MALAT1 promotes proliferation and suppresses apoptosis of glioma cells through derepressing Rap1B by sponging miR-101. J Neurooncol 134: 19-28, 2017.

12. Li H, Yuan X, Yan D, Li D, Guan F, Dong Y, Wang H, Liu X and Yang B: Long non-coding RNA MALAT1 decreases the sensitivity of resistant glioblastoma cell lines to temozolomide. Cell Physiol Biochem 42: 1192-1201, 2017.

13. Yu M, Ohira M, Li Y, Niizuma H, Oo ML, Zhu Y, Ozaki T, Isogai E, Nakamura Y, Koda T, et al: High expression of ncRAN, a novel non-coding RNA mapped to chromosome 17q25.1, is associated with poor prognosis in neuroblastoma. Int J Oncol 34: 931-938, 2009.

14. Zhu Y, Yu M, Li Z, Kong C, Bi J, Li J, Gao Z and Li Z: ncRAN, a newly identified long noncoding RNA, enhances human bladder tumor growth, invasion, and survival. Urology 77: 510.e511-515, 2011.

15. Cayre A, Rossignol F, Clottes E and Penault-Llorca F: aHIF but not HIF-1alpha transcript is a poor prognostic marker in human breast cancer. Breast Cancer Res 5: R223-R230, 2003.

16. Thrash-Bingham CA and Tartof KD: aHIF: A natural antisense transcript overexpressed in human renal cancer and during hypoxia. J Natl Cancer Inst 91: 143-151, 1999.

17. Zhang Q, Matsuura K, Kleiner DE, Zamboni F, Alter HJ and Farci P: Analysis of long noncoding RNA expression in hepatocellular carcinoma of different viral etiology. J Transl Med 14: $328,2016$.

18. Tasharrofi B, Soudyab M, Nikpayam E, Iranpour M, Mirfakhraie R, Sarrafzadeh S, Geranpayeh L, Azargashb E, Sayad A and Ghafouri-Fard S: Comparative expression analysis of hypoxia-inducible factor-alpha and its natural occurring antisense in breast cancer tissues and adjacent noncancerous tissues. Cell Biochem Funct 34: 572-578, 2016.

19. Rossignol F, de Laplanche E, Mounier R, Bonnefont J, Cayre A, Godinot C, Simonnet $\mathrm{H}$ and Clottes E: Natural antisense transcripts of HIF-1alpha are conserved in rodents. Gene 339: 121-130, 2004

20. Span PN, Rao JU, Oude Ophuis SB, Lenders JW, Sweep FC, Wesseling P, Kusters B, van Nederveen FH, de Krijger RR, Hermus AR, et al: Overexpression of the natural antisense hypoxia-inducible factor-1alpha transcript is associated with malignant pheochromocytoma/paraganglioma. Endocr Relat Cancer 18: 323-331, 2011

21. Shen M and Ren X: New insights into the biological impacts of immune cell-derived exosomes within the tumor environment. Cancer Lett 431: 115-122, 2018.

22. Samanta S, Rajasingh S, Drosos N, Zhou Z, Dawn B and Rajasingh J: Exosomes: New molecular targets of diseases. Acta Pharmacol Sin 39: 501-513, 2018.

23. Bahrami A, Aledavood A, Anvari K, Hassanian SM, Maftouh M, Yaghobzade A, Salarzaee O, ShahidSales S and Avan A: The prognostic and therapeutic application of microRNAs in breast cancer: Tissue and circulating microRNAs. J Cell Physiol 233: 774-786, 2018.

24. Lin XJ, Fang JH, Yang XJ, Zhang C, Yuan Y, Zheng L and Zhuang SM: Hepatocellular carcinoma cell-secreted exosomal microRNA-210 promotes angiogenesis in vitro and in vivo. Mol Ther Nucleic Acids 11: 243-252, 2018.

25. Kumata Y, Iinuma H, Suzuki Y, Tsukahara D, Midorikawa H, Igarashi Y, Soeda N, Kiyokawa T, Horikawa M and Fukushima R: Exosome-encapsulated microRNA-23b as a minimally invasive liquid biomarker for the prediction of recurrence and prognosis of gastric cancer patients in each tumor stage. Oncol Rep 40: 319-330, 2018.

26. Azmi AS, Bao B and Sarkar FH: Exosomes in cancer development, metastasis, and drug resistance: A comprehensive review. Cancer Metastasis Rev 32: 623-642, 2013.

27. Lugea A and Waldron RT: Exosome-mediated intercellular communication between stellate cells and cancer cells in pancreatic ductal adenocarcinoma. Pancreas 46: 1-4, 2017.
28. Umezu T, Ohyashiki K, Kuroda M and Ohyashiki JH: Leukemia cell to endothelial cell communication via exosomal miRNAs. Oncogene 32: 2747-2755, 2013

29. Zhao X, Wu X, Qian M, Song Y, Wu D and Zhang W: Knockdown of TGF- $\beta 1$ expression in human umbilical cord mesenchymal stem cells reverts their exosome-mediated EMT promoting effect on lung cancer cells. Cancer Lett 428: 34-44, 2018.

30. Livak KJ and Schmittgen TD: Analysis of relative gene expression data using real-time quantitative PCR and the 2(-Delta Delta C(T)) method. Methods 25: 402-408, 2001.

31. Richards KE, Zeleniak AE, Fishel ML, Wu J, Littlepage LE and Hill R: Cancer-associated fibroblast exosomes regulate survival and proliferation of pancreatic cancer cells. Oncogene 36: 1770-1778, 2017.

32. Ohshima K, Kanto K, Hatakeyama K, Ide T, Wakabayashi-Nakao K, Watanabe Y, Sakura N, Terashima M, Yamaguchi K and Mochizuki T: Exosome-mediated extracellular release of polyadenylate-binding protein 1 in human metastatic duodenal cancer cells. Proteomics 14: 2297-2306, 2014.

33. Orzan F, De Bacco F, Crisafulli G, Pellegatta S, Mussolin B, Siravegna G, D'Ambrosio A, Comoglio PM, Finocchiaro G and Boccaccio C: Genetic Evolution of Glioblastoma Stem-Like Cells From Primary to Recurrent Tumor. Stem Cells 35: 2218-2228, 2017.

34. Godlewski J, Ferrer-Luna R, Rooj AK, Mineo M, Ricklefs F, Takeda YS, Nowicki MO, Salińska E, Nakano I, Lee H, et al: MicroRNA signatures and molecular subtypes of glioblastoma: the role of extracellular transfer. Stem Cell Reports 8: 1497-1505, 2017.

35. Abou-El-Ardat K, Seifert M,Becker K, Eisenreich S,Lehmann M, Hackmann K, Rump A, Meijer G, Carvalho B, Temme A, et al: Comprehensive molecular characterization of multifocal glioblastoma proves its monoclonal origin and reveals novel insights into clonal evolution and heterogeneity of glioblastomas. Neuro Oncol 19: 546-557, 2017.

36. Zhang L, He L, Lugano R, Roodakker K, Bergqvist M, Smits A and Dimberg A: IDH mutation status is associated with distinct vascular gene expression signatures in lower-grade gliomas. Neuro Oncol 20: 1505-1516, 2018.

37. Darlix A, Deverdun J, Menjot de Champfleur N, Castan F, Zouaoui S, Rigau V, Fabbro M, Yordanova Y, Le Bars E, Bauchet L, et al: IDH mutation and 1p19q codeletion distinguish two radiological patterns of diffuse low-grade gliomas. J Neurooncol 133: 37-45, 2017.

38. Yamashita S, Yokogami K, Matsumoto F, Saito K, Mizuguchi A, Ohta $\mathrm{H}$ and Takeshima $\mathrm{H}$ : MGMT promoter methylation in patients with glioblastoma: is methylation-sensitive high-resolution melting superior to methylation-sensitive polymerase chain reaction assay? J Neurosurg: May 4, 2018 (Epub ahead of print).

39. Zhang X, Peng L, Liang Z, Kou Z, Chen Y, Shi G, Li X, Liang Y, Wang F and Shi Y: Effects of aptamer to U87-EGFRvIII cells on the proliferation, radiosensitivity, and radiotherapy of glioblastoma cells. Mol Ther Nucleic Acids 10: 438-449, 2018.

40. Tan SK, Pastori C, Penas C, Komotar RJ, Ivan ME, Wahlestedt C and Ayad NG: Serum long noncoding RNA HOTAIR as a novel diagnostic and prognostic biomarker in glioblastoma multiforme. Mol Cancer 17: 74, 2018.

41. An WG, Kanekal M, Simon MC, Maltepe E, Blagosklonny MV and Neckers LM: Stabilization of wild-type p53 by hypoxia-inducible factor 1alpha. Nature 392: 405-408, 1998.

42. Huang K, Fang C, Yi K, Liu X, Qi H, Tan Y, Zhou J, Li Y, Liu M, Zhang Y, et al: The role of PTRF/Cavin1 as a biomarker in both glioma and serum exosomes. Theranostics 8: 1540-1557, 2018.

43. Treps L,Perret R,Edmond S, Ricard D and Gavard J: Glioblastoma stem-like cells secrete the pro-angiogenic VEGF-A factor in extracellular vesicles. J Extracell Vesicles 6: 1359479, 2017.

44. Zeng AL, Yan W, Liu YW, Wang Z, Hu Q, Nie E, Zhou X, Li R, Wang XF, Jiang T, et al: Tumour exosomes from cells harbouring PTPRZ1-MET fusion contribute to a malignant phenotype and temozolomide chemoresistance in glioblastoma. Oncogene 36: 5369-5381, 2017.

45. Zhao C, Wang H, Xiong C and Liu Y: Hypoxic glioblastoma release exosomal VEGF-A induce the permeability of blood-brain barrier. Biochem Biophys Res Commun 502: 324-331, 2018.

46. Zhao C, Wang H, Xiong C and Liu Y: Hypoxic glioblastoma release exosomal VEGF-A induce the permeability of blood-brain barrier. Biochem Biophys Res Commun 502: 324-331, 2018. 